\title{
Immunology of IDDM
}

\author{
H. Noorchashm ${ }^{1}$, W. K wok ${ }^{2}$, A . R abinovitch ${ }^{3}$, L.C. H arrison ${ }^{4}$ \\ ${ }^{1}$ University of Pennsylvania Hospital, Philadelphia, Pennsylvania, USA \\ ${ }^{2}$ Virginia Mason Research Center, Seattle, Washington, USA \\ ${ }^{3}$ University of Alberta, Edmonton, Canada \\ ${ }^{4}$ The Walter and Eliza Hall Institute, Victoria, Australia
}

Insulin-dependent diabetes mellitus (IDDM) is at the forefront as a paradigm for research on polygenic disorders and on the early diagnosis, prediction and prevention of autoimmune disease. There is growing anticipation that knowledge of the genetic susceptibility and processes of beta-cell destruction, gained over the past two decades, herald an era of targeted therapy for the prevention of IDDM; and without prevention there can be no cure. The genetic dissection of IDDM and the molecular immunology of IDDM are converging on the functional mechanisms by which IDDM susceptibility gene products contribute to disease pathogenesis. This is illustrated by the role of the HLA-DQ8 (3.2) antigen-presenting molecule and its counterpart, $\mathrm{I}-\mathrm{A}^{\mathrm{g} 7}$, in the NOD mouse model of IDDM. Increasing evidence supports the determinant selection model for MHC susceptibility molecules such as HLA-DQ8 (or protective molecules such as DQ6) in which these molecules bind specific peptides that regulate T-cell immunity against pancreatic beta-cells. The appeal of this model is that it identifies not only pathogenic T-cell epitopes but peptides that have potential as immunotherapeutic

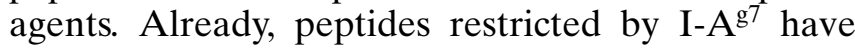
been shown to be immunoregulatory when administered, e.g. transmucosally, in the NOD mouse. The

Participants: J. Todd, Wellcome Trust Centre for Human Genetics, Oxford, UK

C.G. Fathman, Stanford University Medical School, Stanford, California, USA

G. Nepom, Virginia Mason Research Center, Seattle, Washington, USA

M. Christie, King's College School of Medicine, London, UK

A. Lernmark, University of Washington Dept of Medicine, Seattle, Washington, USA

Corresponding author: L.C. Harrison, The Walter and Eliza Hall Institute of Medical Research, Post Office, Royal Melbourne Hospital, Parkville, Victoria 3050, Australia functional consequence of T-cell receptor recognition of peptide-MHC complexes, i.e. the nature of the Tcell response, is determined in the first instance by the peptide antigen itself and the avidity of its interaction.

Given the tremendous advances in the immunology and genetics of IDDM, and the techniques now at our disposal, it is appropriate to further strengthen the marriage between basic research and clinical application.

\section{John Todd: Genetics of IDDM}

IDDM is a polygenic disease. However, family and twin studies have revealed that the "genetic component" contributes less than half of the lifetime risk. Within a panoply of susceptibility genes the major histocompatibility complex (MHC) on human chromosome $6 \mathrm{p} 21$ is the major susceptibility locus, designated IDDM 1 [1]. Polymorphisms in the human HLA-DQB1 and DRB1 loci correlate directly with disease susceptibility (e.g. DQB $1 * 0302$ and DRB1*0401 alleles) or protection (e.g. DQB1*0602 allele), presumably via binding of peptides that regulate anti-islet T-cell responses. The presence of MHC susceptibility alleles is necessary but not sufficient for the development of IDDM. Non-MHC susceptibility loci have also been mapped. Genome-wide linkage analyses have yielded mapping data on up to 15 non-MHC susceptibility loci. None of the nonMHC are as important as the MHC locus but, collectively, are equally as important and necessary for disease development. Two of the non-MHC loci were discussed by Dr. Todd.

The IDDM 2 locus is located at the $5^{\prime}$ end of the insulin gene on chromosome 11. The IDDM 2 locus is polymorphic for a variable number of tandem repeats (VNTR) within the promoter 600 bp upstream 
of the insulin ATG codon. The VNTR has two main classes of alleles. The class III allele has more than 200 copies of the repeat and is dominantly protective (decreases risk of IDDM by $70 \%$ ). The class I allele has 30-40 copies of the repeat and is recessive. The VNTR can influence proinsulin gene expression in a tissue-specific fashion $[2,3]$. In the pancreas, the class III allele is associated with a lower level of proinsulin expression, whereas in the thymus it is associated with a higher level of proinsulin expression. It is speculated that a peptide from proinsulin/insulin as a self-antigen bound to MHC in thymus tolerizes proinsulin-reactive $\mathrm{T}$ cells. This supports evidence from Dr. Harrison's group that proinsulin is a primary antigen in IDDM, viz à vis the finding of proinsulin peptide-reactive $\mathrm{T}$ cells in at-risk first-degree relatives and the absence of insulitis and diabetes in NOD mice transgenic for proinsulin on a class II MHC promoter.

The murine IDDM 3 locus on chromosome 3 has now been mapped in detail. Studies with congenic mice have located IDDM 3 to a $0.15 \mathrm{cM}(600 \mathrm{~kb})$ region which contains the interleukin (IL)-2 gene. Other studies with congenic mice reveal that NOD mice secrete less IL-2 compared to the diabetes-resistant strain. Reciprocal congenic strains of NOD and $\mathrm{B} 6 / \mathrm{B} 10$ indicate that inter-strain variation in IL-2 production maps to the same congenic interval as IDDM 3 and IL-2 (L. Wicker, L. Peterson and P. Lyons, unpublished data). Amino acid polymorphism has been observed in the first exon of the IL2 gene. NOD mice have a proline substitution and a Serine, serine, proline, threonine (SSPT) insert compared to the diabetes-resistant strain. The proline substitution and the SSPT insert generate a proline, glutamate, serine, threonine (PEST) sequence, which is known to influence protein stability. Both NOD and diabetes-resistant strains have similar levels of IL-2 mRNA (P. Lyons and C. Lord, unpublished data). It is speculated therefore that the PEST sequence in the IL-2 of the NOD mouse destabilizes the protein. These data are in concert with previous reports that IL-2 levels are lower in human IDDM and that IL-2 is essential for the maintenance of self-tolerance and the prevention of autoimmunity. They are consistent with a model in which the IDD M 3/IL-2 allele in NOD mice encodes a relative deficiency of IL-2 and, conversely, in which the diabetes-resistant allele of the C57BL/6 strain encodes higher levels of IL-2 which drastically reduce the diabetogenic potential of the NOD mouse background [4].

There is a great need to source cell-DNA from many more IDDM families in different ethno-geographic groups in order to identify IDDM susceptibility genes. DNA repositories should be shared between investigators to improve statistical confidence and validate the conclusions of smaller studies. The autoimmune phenotype is the outcome of the interplay of many genes and epigenetic factors interacting with environmental factors over time. Complex genetic and epigenetic interactions in a polygenic disorder mean that it can be difficult to dissect the relative contributions of individual genes. For example, the relative risk associated with the recently described IDDM 13 locus was shown to be significantly higher when the effect of MHC susceptibility genes was removed [5]. Furthermore, in polygenic disorders environmental co-factors may be multiple and differ from one ethno-geographic group to another, even one individual to another, despite the apparent homogeneity of the clinical syndrome. Thus, the finding in IDDM genetics that the relative risk for some nonMHC loci varies considerably in different centres may not be unexpected.

\section{Gerald Nepom: HLA-DQ genes, function and regulation}

HLA DQ3.2, the strongest IDDM-associated HLA molecule, is encoded by the DQA $1 * 0301 /$ DQB $1 * 0302$ genes, and has an interesting and unique peptide binding motif $[6,7]$. Four amino acid positions in a peptide, positions 1, 4, 6 and 9, appear to be crucial for binding. Although specific amino acid residues at positions 1 and 6 can also bind to other DQ molecules, the amino acid side chains at positions 4 and 9 that are permissive for binding to DQ3.2 are more limited. Peptides which contain a negatively charged amino acid, Asp(D) or Glu(E), at position 9 bind well to DQ3.2. The structural basis for this binding property is the increased avidity of interaction between peptides with a negative charge in position 9 and charged residues in the class II molecule. When codon 57 of the class II $\beta$ chain, which commonly encodes an Asp, instead encodes a small aliphatic residue such as in DQ3.2, peptides with a negative charge at position 9 bind more efficiently. The most likely interpretation is that a positively charged Arg residue on the DQ $\alpha$ chain which forms an ion pair with the Asp57 residue on the $\beta$ chain, pairs instead with the side chain contributed by the peptide at a homologous position.

Although direct binding studies delineate the preferred structural motifs for binding to DQ3.2, the relationship between these motifs and IDDM susceptibility is not as clear. For example, the DQ3.3 molecule, which is associated with IDDM in Oriental populations, contains an Asp57 residue, which markedly skews its peptide motif pattern relative to DQ3.2. Another confounding issue relates to the specific $\alpha$ and $\beta$ chain combinations present on disease-associated molecules. While the DQA1*0301/DQB1*0302 dimer accounts for disease susceptibility on DR4 haplotypes, it has often been suggested that the trans encoded heterodimer, 
DQA $1 * 0501 / \mathrm{DQB} 1 * 0302$, is also associated with susceptibility. Structural studies have shown, however, that the peptide binding motifs for this trans heterodimer are quite different than for the traditional DQ3.2 dimer. This suggests that more than one MHC-peptide context may be pathogenic.

An intriguing facet of IDDM susceptibility is the protective effect of the DR2 haplotype containing DRB1*1501 and DQB1*0602. Not only is this class II haplotype rarely found in diabetic patients, it appears to confer dominant protection from diabetes in the sense that individuals with one IDDM susceptibility haplotype, DR4-DQB1*0302, are not susceptible to IDDM if heterozygous for the protective DR2 haplotype. One of the models proposed to account for this dominant protection is based on peptide binding by protective and susceptible class II alleles. In this model, it is postulated that peptides which bind an IDDM susceptibility molecule such as DQ3.2 can also bind a class II molecule from the protective DR2 haplotype. A straightforward competitive binding relationship is envisioned, wherein high affinity binding by protective class II molecules effectively prevents binding of peptide to the susceptible HLA molecules.

Not all peptides which bind class II molecules carry allele-specific motifs. So-called "promiscuous" peptides have been described which are polyspecific. Obviously, if IDDM pathogenic peptides belong to this family of indiscriminate MHC binders, then analysis of allele-specific motifs associated with susceptibility is unlikely to provide much insight into the identity of triggering antigens. Given our current knowledge of class II structure and function, however, it seems most reasonable to assume that the high degree of allelic specificity with disease susceptibility indicates a high degree of allelic specificity for peptide determinant selection. Thus, a major goal of the analysis of peptide motifs in IDDM susceptibility molecules is to facilitate the prediction and testing of specific antigenic peptides which account for the early autoimmune activation process.

Dr. Nepom also discussed the importance of binding motifs in determining the outcome of T-cell recognition. Thus, a DQ3.2-restricted T-cell clone recognizes the HSV 433-445 peptide when presented by a DQ3.2 antigen-presenting cell (APC). The HSV 433-445 peptide has a negatively charged residue at position 9. The T-cell clone cannot recognize the 433-445 peptide when presented by a DQ3.3 APC. The DQ3.3 molecule differs from the DQ3.2 molecule only in having an Asp at position 57 of the $\beta$ chain. Interestingly, the same T-cell clone recognizes the 433-445 10A peptide, with an Ala substitution at position 9, when presented by a DQ3.3 but not by a DQ3.2 APC. These studies indicate that knowledge of binding motifs IDDM can facilitate prediction of an antigenic peptide.
Signalling by the class II MHC-peptide complex through the T-cell receptor is not a simple event. In addition to modulation by cytokine stimuli and accessory molecule recognition, the T-cell receptor can be differentially triggered by subtle structural changes involving MHC-peptide recognition itself. For example, the same peptide presented on different class II molecules can trigger $T$ cells along two alternative differentiation pathways. As another example, single amino acid changes in the peptide, or in the MHC molecule that influence peptide conformation, can cause the MHC-peptide complex to antagonize or only partially activate a $\mathrm{T}$ cell.

Levels of expression of class II MHC-peptide complexes can similarly determine differential T-cell outcomes. In this density-dependent threshold phenomenon, high levels of MHC-peptide complexes trigger $\mathrm{T}$ cells along differentiation pathways distinct from those of T cells triggered by low density MHC-peptide complexes.

Structural differences between DQ alleles may also account for differences in their intrinsic stability [8]. The DQ3.2 molecule is relatively unstable at neutral $\mathrm{pH}$ compared to the DQ3.1 molecule, which only differs from DQ3.2 at position 13,26, 45 and 57 in the first exon. DQ molecules also exhibit differences in $\mathrm{pH}$ optimal for peptide binding. DQ3.2 has an optimal of 5.5, compared to 6.5 for DQ3.1. Both intrinsic stability and $\mathrm{pH}$ dependency of peptide binding can influence the level of MHC-peptide complexes on the cell surface and thus T-cell activation.

Differences in regulation of transcription could also influence the levels of MHC-peptide complexes on the cell surface. Dr. Nepom described DNA polymorphism in the promoter region of the different DQ alleles. The DQ3.2 allele has a TG dinucleotide at the $5^{\prime}$ end of the $\mathrm{X}$ box, absent in the DQ3.1 allele. The DQ3.1 promoter is also a much stronger promoter compared to the DQ3.2 promoter. The transcription factor YY-1 interacts with the DQ promoter region. It is speculated that binding of YY-1 in the absence of TG enhances transcription while binding in the presence of TG reduces transcription.

Examples of how the T-cell response is influenced by alterations in the quantity or quality of MHC-peptide expression illustrate the potential for important functional consequences of disease-associated class II MHC determinant selection. Where density is a prime determinant of outcome, factors which influence levels of expression of class II MHC molecules are likely to have a significant impact. This densitydependent model integrates differences in levels of expression, possibly influenced by transcriptional variation, and provides a direct mechanism by which the peptide-competition model results in a functional distinction between susceptibility and protective Tcell outcomes. 


\section{Garrison Fathman: T-cell responses to self antigens in NOD mice}

Just as the majority of Caucasoids with IDDM are homozygous for expression of the $\mathrm{DQ} \beta 57$ non-Asp alleles, similarly NOD mice, a model of IDDM, are homozygous for a non-Asp amino acid, serine, at position 57 of their $\mathrm{DQ} \beta$ counterpart, I-A $\beta^{\mathrm{g} 7}$.NOD F1 mice, heterozygous for non-Asp at position 57, have circa approximately $3 \%$ incidence of diabetes; however, in F2 mice from various breedings greater than $95 \%$ of those that develop diabetes are nonAsp homozygotes. The NOD mouse therefore serves as a paradigm for $\mathrm{MHC}$-associated autoimmune disease. NOD mice exhibit a wider range of autoimmune phenomena, including lymphocytic infiltration of the thyroid, salivary and adrenal glands, the bowels and the testes. $T$ cells in these tissues exhibit a range of T-cell receptor specificities and do not seem to be clonally related. MHC-associated autoimmune disease is assumed to reflect presentation by APCs of disease-associated autoantigen peptides. However, it is difficult to see how this peptide binding-determinant mechanism is consistent with a requirement for non-Asp homozygosity in both human and mouse IDDM. This puzzle led to the hypothesis that a requirement for MHC homozygosity could reflect defective thymic selection of $\mathrm{T}$ cells resulting in a T-cell repertoire containing a high frequency of polyspecific high-affinity autoreactive $\mathrm{T}$ cells. Dysregulated peripheral expansion of autoreactive T-cell clones directed towards particular tissues might then occur due to a variety of immune perturbations.

To test this hypothesis NOD mice were primed with self peptides, from myoglobin and TCRVb8.2, in complete Freund's adjuvant (CFA). CD4 ${ }^{+} \mathrm{T}$ cells from primed mice proliferated to these self-antigens endogenously processed and presented by APCs from unimmunized NOD mice. This phenomenon, termed "autoproliferation" [9], was not observed in non-NOD strains. When queried whether autoproliferation was found in non-NOD strains that expressed $\mathrm{I}-\mathrm{A}^{\mathrm{g} 7}$, for example Biozzi $\mathrm{AB} / \mathrm{H}$ mice which develop experimental autoimmune encephalomyelitis, Dr. Fathman replied that Biozzi mice had not been tested but that NOR mice ( $\left.\mathrm{I}-\mathrm{A}^{\mathrm{g} 7}\right)$ exhibited autoproliferation. NOD (I-A $\left.{ }^{\mathrm{g} 7}\right)$, NOD I-A $\mathrm{A}^{\mathrm{k}}$ MHC congenic mice $\left(\mathrm{I}-\mathrm{A}^{\mathrm{k}}\right.$ only) and NOD $\mathrm{I}-\mathrm{A}^{\mathrm{k}}$ transgenic mice $\left(\mathrm{I}-\mathrm{A}^{\mathrm{k}}\right.$ and $\mathrm{I}-\mathrm{A}^{\mathrm{g} 7}$ ) were used to examine the role of MHC class II in autoproliferation. Autoproliferative T cells could be generated in NOD I-A ${ }^{\mathrm{k}}$ transgenic but not in NOD I-A ${ }^{\mathrm{k}}$ congenic mice. Thus, autoproliferative $\mathrm{CD}^{+}{ }^{+} \mathrm{T}$ cells from NOD I-A ${ }^{\mathrm{k}}$ transgenic mice were exclusively restricted by $\mathrm{I}-\mathrm{A}^{\mathrm{g} 7}$. The implication therefore is that lack of autoproliferative T cells from $\mathrm{I}-\mathrm{A}^{\mathrm{k}}$ congenic mice is due to a thymic negative selection of autoreactive T cells.
TCR V $\beta 8.2$ peptide primed CD $4^{+}$T cells from I$\mathrm{A}^{\mathrm{k}}$ transgenic NOD mice were only capable of autoproliferating when stimulated with APC from NOD mice and $\mathrm{I}^{-\mathrm{A}^{\mathrm{k}}}$ transgenic NOD mice, but not from $\mathrm{I}^{-} \mathrm{A}^{\mathrm{k}}$ congenic NOD mice. Thus, autoproliferative $\mathrm{T}$ cells from the I-A ${ }^{k}$ transgenic NOD mice are restricted by the NOD I-A $\mathrm{A}^{\mathrm{g} 7}$. These observations support the model of "altered" thymic selection of I-A $\mathrm{A}^{\mathrm{g} 7}$-restricted $\mathrm{T}$ cells in which autoreactive $\mathrm{T}$ cells escape thymic negative selection. It was suggested that structural instability and a poor ability of $\mathrm{I}-\mathrm{A}^{\mathrm{g} 7}$ to bind peptides might be responsible for "altered" thymic selection in NOD mice, as proposed by Carrasco-Marin et al. [10]. The latter reported that soluble I- $\mathrm{A}^{\mathrm{g} 7} \alpha \beta$ molecules were unstable and virtually undetectable by SDS-PAGE, and that this decreased stability correlated with decreased cell surface expression. On the other hand, Harrison et al. [11] have recently shown that purified $\mathrm{I}-\mathrm{A}^{\mathrm{g} 7}$ is stable and retains peptide binding function in vitro. As discussed by Dr. Nepom above, in contrast to the non-susceptibility DQ3.1 molecule which is stable in a wide $\mathrm{pH}$ gradient, the IDDM susceptibility molecule, DQ3.2, is less stable at neutral $\mathrm{pH}$ than at acidic $\mathrm{pH}$. However, it should be noted that MHC-peptide binding occurs in the acidic milieu ( $\mathrm{pH}$ 5-5.5) of the endosomes, at which both DQ3.1 and 3.2 are stable.

By employing a differential avidity model of thymic selection [12], structurally and functionally defective $\mathrm{I}-\mathrm{A}^{\mathrm{g} 7}$ could explain the propensity for the escape of autoreactive T cells into the periphery. Current models suggest that thymic cortical epithelial cells bearing self-MHC class II molecules plus self-peptides allow positive selection of $\mathrm{CD} 4^{+} \mathrm{T}$ cells of low to high avidity. Subsequently, thymic bone marrow-derived MHC class II positive cells bearing self-peptides, delete high avidity self-reactive $\mathrm{CD} 4^{+} \mathrm{T}$ cells (negative selection). Both positive and negative selections occur at some pre-selected threshold of MHC:T-cell avidity. If overall MHC:T-cell avidity is proportional to [MHC:peptide] $\times\left[\right.$ TCR affinity], diminished $\mathrm{I}-\mathrm{A}^{\mathrm{g} 7}$ self-peptide binding could lower the effective concentration of the MHC:peptide complex (i.e. effective ligand dose) necessitating a compensatory increase in T-cell receptor affinity to achieve an equivalent avidity threshold for positive (as well as negative) selection. Such a shift might result in the escape into the periphery of T cells with high affinity receptors for self.

Dr. Fathman concluded that I-A $\mathrm{A}^{\mathrm{g} 7}$-dependent altered thymic selection could explain the appearance of autoreactive $\mathrm{T}$ cells and the diverse autoimmune manifestations in NOD mice. In discussion, Dr. Harrison added that their reported finding of T-cell reactivity to certain IDDM autoantigen peptides, e.g. proinsulin 24-36, in both 'at-risk' relatives and HLA-matched control subjects would support the view that autoimmune disease-associated MHC molecules are permissive for T-cell autoreactivity. 


\section{Michael Christie: The elusive autoantigen}

Over the past 10 years, major efforts have been made to identify primary target autoantigens that drive autoimmune-mediated beta-cell destruction in IDDM. Analysis by a range of biochemical and molecular techniques in both human IDDM and animal models has led to a long list of potentially important antigens described in the literature. These range from relatively tissue-restricted proteins such as insulin, glutamic acid decarboxylase (GAD) and the tyrosine phosphatase-like protein, IA-2, to glycolipids such as ganglioside Gm2-1, and also include ubiquitous cell components such as tubulin and actin. (Pro)insulin is the only islet antigen that is absolutely beta-cell specific. One is left with the impression that virtually any component expressed by the pancreatic beta cell can be the target of increased immune reactivity in IDDM.

The long list of candidate antigens contrasts with the relatively simple pattern of proteins detected originally by Baekkeskov and Lernmark in the early 1980 s. These predominantly single bands of about 64 $\mathrm{kDa}$, specifically precipitated by antibodies in sera from IDDM patients, suggested that there may be a single primary target autoantigen. However, it has now become clear that the $64 \mathrm{kDa}$-antigen identified then is in fact composed of at least three distinct molecular species, namely GAD and two tyrosine phosphatase-like proteins, IA-2 $\alpha$ (or ICA512) and phogrin (or IA- $2 \beta$ ). Furthermore, the SDS-PAGE was not tailored to detect low mol. wt. antigens such as insulin or proinsulin. There is also evidence to suggest that the experimental conditions used to maintain islets or islet cell lines can influence antigen expression and that novel autoantigens can be detected by manipulation of culture conditions. For example, expression of a $38 \mathrm{kDa}$ glycoprotein (GLIMA) is strongly influenced by unidentified factors in fetal calf serum used to maintain cells in culture. It was stated that antibodies to neither GAD nor IA-2 were detected in NOD mice by immunoprecipitation of those proteins synthesized and biosynthetically labelled in an in vitro transcription-translation assay. However, both the Maclaren and Harrison laboratories have reported the finding of antibodies to native GAD in NOD mice.

Apart from (pro)insulin, GAD and IA-2, the evidence is weak for other antigens in IDDM. It would be helpful to adopt a set of clinical and experimental criteria against which the authenticity of a candidate autoantigen could be evaluated and standardised. GAD, insulin and IA-2 all contribute to islet cell antibody (ICA) detected by immunofluorescence staining with patient sera on frozen sections of pancreas, because these proteins block ICA staining. This blocking is incomplete, implying that other islet cell antigens also contribute to ICA reactivity. There is a clear need to determine the nature of the remaining ICA antigen(s).

Recent work has examined whether the two closely related tyrosine phosphatases, IA- $2 \alpha$ and $\beta$, are independent antigens in IDDM. Blocking of autoantibody binding with recombinant proteins indicates that most of the reactivity to I-A $2 \beta$ (phogrin) can be accounted for by cross-reactive epitopes on IA- $2 \alpha$. However, a significant number of non-diabetic subjects with polyendocrine autoimmunity have been found to have exclusively I-A2 $\beta$-specific antibodies. This finding, which is very rare in patients with recent-onset IDDM, suggests that a humoral response to I-A $2 \beta$ can develop independently to that to IA- $2 \alpha$.

The mere detection of an antibody specificity in serum says little about the role of the autoantigen in the disease process. There is considerable interest in examining the natural history of autoimmunity in the pre-diabetic period. In collaboration with Annette Ziegler in Munich, Dr. Christie and colleagues [13] showed that antibodies to GAD, IA-2 and insulin can all develop in the first 3 years of life with no discernible differences in order of appearance. There was no evidence for a single target autoantigen in these studies. In most cases, antibody levels were remarkably stable. However, prospective studies in older IDDM relatives have shown decreases in antibody levels, and even disappearance of antibodies, before the onset of IDDM [14]. Thus, antibody status at onset of clinical IDDM, frequently taken as a measure of marker sensitivity, does not necessarily reflect antibody status during the pre-diabetic period. Establishing when specific antibodies appear and disappear is particularly important in determining the appropriate age to conduct antibody screening.

IDDM is a T-cell mediated disease and autoantigen-specific $\mathrm{T}$ cells are obvious targets for immune intervention therapy. Assays for these T cells are likely to be the most valid markers of pre-clinical disease progression and for assessing the effectiveness of intervention therapies. It is therefore of considerable importance to characterize autoantigen-specific Tcell responses. Such studies may provide clues as to the relevance of individual antigens in the disease process. Clearly, the T cells of most interest are located within the inflammatory milieu of the islet, but these are virtually impossible to access in humans. However, the relevant $\mathrm{T}$ cells may be present, albeit at much lower frequency, in peripheral blood of prediabetic subjects. Increased T-cell proliferation to recombinant (pro)insulin or GAD has been reported, but assay design and quality control are suboptimal and need to be addressed by international workshops. Dr. Christie described T-cell studies with recombinant IA-2 expressed in Escherichia coli. T-cell responses in IDDM patients were increased compared to control subjects, but this was due predominantly to contaminants in the antigen preparation. 
Therefore, T-cell lines were generated from IDDM patients by repeated cycles of stimulation of peripheral blood mononuclear cells with IA-2 and IL-2. Lines with varying specificity to IA-2 were obtained from IDDM patients but not from control subjects. E. coli-derived heat shock protein (hsp) 60 appears to be a major component of the recombinant antigen preparation that contributes to proliferation of $T$ cells. However, some lines also responded to IA-2 synthetic peptides and showed HLA-restricted responses to specific regions of the IA-2 molecule. Generation of T-cell lines to the recombinant autoantigen may therefore be a valuable means of characterizing the T-cell response in IDDM. Further work is clearly needed to improve the quality of autoantigen preparations, as well as the assays themselves, so that T-cell reactivity to autoantigens can be measured reliably. Åke Lernmark: Immunology of IDDM - present and the
future

The understanding of IDDM in humans has been improved by epidemiological studies including the use of registries, the identification of several autoantigens, development of standardized autoantibody assays using recombinant antigens to predict diabetes, analysis of human T-cell proliferation in response to autoantigens, continuing HLA association and linkage studies and genome-wide scanning of affected sibpairs to detect loci linked to IDDM. In parallel, immunointervention studies in animal models have shortened the time between experimental observations and their implementation in controlled clinical trials.

Genetic analysis of population-based patients and control subjects has demonstrated that HLA genes are the most important in determining genetic risk for IDDM. On the HLA-DR4;DQ8 haplotype, DQ8 (3.2) is more important than DR4. On the DR3;DQ2 haplotype, it has not been possible to resolve which allele is most important. It is also observed that DQ6 is strongly negatively associated with IDDM. Susceptibility or protection by HLA is, however, "leaky" as the strength of the association decreases significantly with age. It is a testable hypothesis that genes referred to as modifier genes may affect either immune responses, beta-cell function or both. The well-known environmental factors implicated in triggering IDDM are virus infections causing latent immunity (coxsackie, rubella) or betacell lysis (mumps), beta-cell toxic agents (such as food items with nitrosamines) and other more speculative factors that might, for example, alter intrinsic sensitivity to apoptosis leading to beta-cell death.

Two recent studies, from Finland [15] and Sweden [16], investigated children who had developed diabetes between 1 and 15 years of age. The availability of serum samples from the mothers at the time of birth meant that maternal sera could be analysed for antiviral antibodies. An increased frequency of enterovirus antibodies was detected in mothers of children who developed IDDM later in life. The conclusion was that gestational infection is a major risk factor for the development of IDDM, as demonstrated in the 1970 s for congenital rubella. It is unclear why the time to onset is variable. A fetal-initiating event in some children may be associated with an early age at onset, while in others islet autoimmunity is induced but may not result in clinical diabetes until many years later. Hence, it was of interest to determine the number of children with islet autoantibodies in cord blood who developed diabetes during 15 years' follow-up. Preliminary findings suggest that about $20 \%$ of such children had been positive for ICA, GA$\mathrm{D} 65 \mathrm{Ab}$ or IAA in cord blood. It is attractive, therefore, to consider the pathogenesis of IDDM in two stages. In Stage I an initiating event influenced by environmental factors debilitates the beta cell. Stage I may occur in utero, or later in life. Stage II is an HLA-dependent disease process leading to accelerated beta-cell destruction via a specific anti-beta-cell autoimmune response. Stage II is rapid in children, but slower in adults.

Recent population-based analyses of people who develop IDDM between the ages of 0 to 35 years of age, demonstrate that the relative risk of the most strongly associated genotype, HLA-DQ2,8 decreases with age. Similarly, there is diminishing protection of DQ6 allele with age. Furthermore, analyses of autoantibodies demonstrates that diagnostic sensitivity decreases with increasing age for IAA, IA-2Ab and ICA, but not for GAD65Ab. This is reflected by the considerable difficulty in classifying patients with diabetes in this age group. Diagnosis of diabetes is based on blood glucose measurements, whereas classification follows a set of recommended clinical criteria. Among 15-34-year-old patients, about $20 \%$ are typically classified as non-insulin-dependent diabetes (NIDDM) or unclassifiable diabetes; as many as 30$50 \%$ may have one or more islet cell antibodies consistent with the requirement (eventually) for insulin treatment. This late or slow onset IDDM has also been termed latent autoimmune diabetes in the adult (LADA), type 1.5 or type 3 diabetes. Its recognition is important for intervention-prevention strategies.

Primary intervention implies that all individuals are treated. In one sense this is analogous to vaccination against infection. The term vaccination can be used, but not in the traditional sense, to describe some ways of preventing autoimmune disease. These include mucosa-mediated immune tolerance to an autoantigen delivered orally or intra-nasally, or other means of exposing an individual to the autoantigen to induce a state of tolerance to 'self'. Secondary 
intervention implies screening for susceptibility genes (about $50-70 \%$ of northern Europeans would be excluded from treatment if presence of DQ2 and/or DQ8 and absence of DQ6 were the inclusion criteria), autoantibody markers or both. The European Nicotinamide Diabetes Intervention Trial (ENDIT), the Diabetes Prevention Trial-1 (DPT-1) with injectable or oral insulin, and the Melbourne Intra-nasal Insulin Trial (MINIT) are secondary intervention trials. Future trials may include other approaches to either immune or metabolic intervention in different at-risk groups. An interesting group are adults with diabetes, often classified as NIDDM but with autoantibody markers and low C-peptide levels (LADA, type 1.5 or type 3 diabetes). Rare patients, such as those severely ill with Stiff Man syndrome and diabetes, may benefit from specific antibody adsorption via plasmapheresis.

Tertiary intervention is currently used in people with long term diabetes who require kidney transplantation. Segmental pancreas grafting is reasonably successful and islet grafting, although still a research procedure, has had limited success. The emergence in many patients of insulitis or the re-appearance of GA$\mathrm{D} 65 \mathrm{Ab}$ or ICA suggests that the loss of graft function would, at least in part, be due to recurrence of disease.

In summary, a rapidly increasing learning curve of knowledge of immunology has heralded novel approaches to early diagnosis, prediction and intervention-prevention of IDDM. Interaction and collaboration among researchers to standardize autoantibody assays and glucose tolerance tests, and to scan the human genome using shared family DNA-cell banks, has been highly successful. The identification of multiple susceptibility genes in IDDM is not a setback but has provided novel insights into the complexity of disease susceptibility. It will be of critical importance to expand the collection of DNA and cell lines from families to improve the precision of data and enable cloning of IDDM genes. This is important for secondary intervention trials to increase sensitivity and specificity of the inclusion criteria used to randomize individuals at risk.

\section{Future directions and recommendations}

\section{Specific}

- Further characterize IDDM-associated MHC (HLA) molecules according to function, i.e. their ability to bind peptides from IDDM autoantigens, and thereby identify peptides for potential diagnostic and immunotherapeutic applications.

- Define criteria for the pathogenicity of an autoantigen.

- Establish international committees/workshops on the design and standardization of T-cell assays in IDDM.
- Support prospective studies, e.g. in babies and infants, to identify environmental trigger factors and the sequence of autoimmune responses.

- Develop animal models of the human immune system, i.e. "humanized" mice, that express human immune genes introduced transgenically or as lymphoid stem cells, to be made generally available to researchers.

- Support the collection and interchange of cellDNA from IDDM families on all continents and networking between investigators to identify further susceptibility and protective genes.

- Support the development and availability of genetically inbred standard animals (e.g.pigs) for transplantation (including gene therapy) studies. Much experimentation in the transplant immunology area is and will be irrelevant to humans unless and until such animals are used.

- Support establishment of a network of designated clinical trials centres in the Americas, Europe and Australasia, analogous to the oncology trial centres, that would conjoin a critical mass of interdisciplinary experts (clinicians, scientists, epidemiologists, statisticians, research nurses, psychologists and others) to direct intellectual and material resources toward the design and execution of clinical trials for intervention and/or prevention in IDDM. Apart from pooling of expertise and resources (e.g. protocols, ethics applications including plain language statements' for trial subjects, etc), this would facilitate development of new trial designs, e.g. for shorter term evaluation of therapy, evaluation of surrogate markers for monitoring therapy, and evaluation of multiple potential therapies.

\section{G eneral}

- JDFI could play a role as an 'honest broker' between commercial interests and academic researchers to facilitate the availability of resources to meet its goals, e.g. of quality controlled, inbred pigs for gene therapy and/or transplantation research.

- JDFI could attract into IDDM research young researchers in particular who are trained in basic sciences by mentors, laboratories and institutions not involved in IDDM research.

\section{References}

1. Todd JA, Farrall M (1996) Panning for gold: genome-wide scanning for linkage in type 1 diabetes. Hum Mol Genet 5: 1443-1448

2. Vafiadis P, Bennett ST, Todd JA et al. (1997) Insulin expression in human thymus is modulated by INS VNTR alleles at the IDDM2 locus. Nature Genet 15: 289-297

3. Pugliese A, Zeller M, Fernandez A Jr et al. (1997) The insulin gene is transcribed in the human thymus and 
transcription levels correlate with allelic variation at the INS VNTR-IDDM2 susceptibility locus for type 1 diabetes. Nature Genet 15: 293-297

4. Denny P, Lord CJ, Hill NJ et al. (1997) Mapping of the IDDM locus Idd 3 to a 0.35 -cM interval containing the interleukin-2 gene. Diabetes 46: 695-700

5. Morahan G, Huang D, Tait BD, Colman PG, Harrison LC (1996) Markers on distal chromosome $2 \mathrm{q}$ linked to insulin-dependent diabetes mellitus. Science 272: 18111813

6. Kwok WW, Domeier ME, Raymond FC, Byers P, Nepom GT (1996) Allele-specific motifs characterize HLA-DQ interaction with a diabetes-associated peptide derived from glutamic acid decarboxylase. J Immunol 156: 2171-2177

7. Kwok W, Domeier M, Johnson M, Nepom GT, Koelle D (1996) HLA-DQB1 codon 57 is critical for peptide binding and recognition. J Exp Med 183: 1253-1258

8. Buckner J, Kwok WW, Nepom B, Nepom GT (1996) Modulation of HLA-DQ binding properties by differences in class II dimer stability and $\mathrm{pH}$-dependent peptide interactions. J Immunol 257: 4940-4945

9. Ridgway WM, Fassò M, Lanctot A, Garvey C, Fathman CG (1996) Breaking self-tolerance on nonobese diabetic mice. J Exp Med 183: 1657-1662

10. Carrasco-Marin E, Shimizu J, Kanagawa O, Unanue ER (1996) The class II MHC I-A ${ }^{\mathrm{g} 7}$ molecules from non-obese diabetic mice are poor peptide binders. J Immunol 156 : $450-458$

11. Harrison LC, Honeyman MC, Trembleau S et al. (1997) A peptide-binding motif for I-A $\mathrm{A}^{\mathrm{g} 7}$, the class II major histocompatibility complex (MHC) molecule of NOD and Biozzi AB/H mice. J Exp Med 185: 1013-1022

12. Kim DT, Rothbard JB, Bloom DD, Fathman CG (1996) Quantitative analysis of T-cell activation. J Immunol 156: 2737-2742

13. Roll U, Christie MR, Fuchtenbusch M, Payton MA, Hawkes CJ, Ziegler AG (1996) Perinatal autoimmunity in offspring of diabetic parents: the German multicentre 'BABY-DIAB' study. Detection of humoral immune responses to islet antigens in early childhood. Diabetes 45: 967-973

14. Christie MR, Roll U, Payton MA, Hatfield ECI, Ziegler AG (1997) Validity of screening for individuals at risk for type 1 diabetes by combined analysis of antibodies to recombinant proteins. Diabetes Care 20: 965-970

15. Hyöty H, Hiltunen P, Knip M et al. (1995) A prospective study of the role of coxsackie B and other enterovirus infections in the pathogenesis of IDDM. Childhood Diabetes in Finland (DiMe) Study. Diabetes 44: 652-657

16. Dahlquist G, Ivarsson S, Lindberg B, Forsgren M (1995) Maternal enteroviral infection during pregnancy as a risk factor for childhood IDDM. Diabetes 44: 408-413 